PROCEEDINGS OF THE

AMERICAN MATHEMATICAL SOCIETY

Volume 137, Number 2, February 2009, Pages 663-668

S 0002-9939(08)09556-7

Article electronically published on September 5, 2008

\title{
EXTRAPOLATION SPACES FOR $C$-SEMIGROUPS
}

\author{
MIAO LI AND QUAN ZHENG
}

(Communicated by Joseph A. Ball)

\begin{abstract}
Let $\{T(t)\}_{t>0}$ be a $C$-semigroup on $X$. We construct an extrapolation space $X_{s}$, such that $X$ can be continuously densely imbedded in $X_{s}$, and $\left\{T_{s}(t)\right\}_{t \geq 0}$, the extension of $\{T(t)\}_{t \geq 0}$ to $X_{s}$, is strongly uniformly continuous and contractive. Using this enlarged space, we give an answer to the question asked in [M. Li, F. L. Huang, Characterizations of contraction $C$-semigroups, Proc. Amer. Math Soc. 126 (1998), 1063-1069] in the negative.
\end{abstract}

\section{INTRODUCTION}

Let $X$ be a Banach space, $\mathbf{B}(X)$ the space of all bounded linear operators on $X$, and $C$ an injective operator in $\mathbf{B}(X)$. A family of linear bounded operators $\{T(t)\}_{t \geq 0} \subset \mathbf{B}(X)$ is called a $C$-semigroup if $T(\cdot)$ is strongly continuous and $T(0)=$ $C, T(t+s) C=T(t) T(s)$ for $t, s \geq 0$. Its generator, $A$, is defined by

$$
A x=C^{-1}\left(\lim _{t \rightarrow 0} \frac{T(t) x-C x}{t}\right)
$$

with maximal domain.

A $C$-semigroup $\{T(t)\}_{t \geq 0}$ is bounded if there is a constant $M>0$ such that $\|T(t)\| \leq M$ for all $t \geq 0$ and is a contraction $C$-semigroup if $\|T(t) x\| \leq\|C x\|$ for all $x \in X$ and $t \geq 0$.

It is natural that all bounded $C_{0}$-semigroups are strongly uniformly continuous, while for $C$-semigroups this is far from obvious. However, we show in this paper that for every bounded $C$-semigroup $\{T(t)\}_{t \geq 0}$ on $X$, an extrapolation space $X_{s}$ can be constructed such that the extension of $\{T(t)\}_{t \geq 0}$ to $X_{s},\left\{T_{s}(t)\right\}_{t \geq 0}$, is a strongly uniformly continuous contraction $C_{s}$-semigroup on $X_{s}$, where $C_{s}$ is the extension of $C$ to $X_{s}$. Our extrapolation space is smaller than the one given by deLaubenfels ([1, 2]).

Moreover, we take up the open problem asked in [4]. The question was: Suppose that $A$ is the generator of a contraction $C$-semigroup on $X$. Does there exist a restriction of $A, A^{\prime}$, which is the generator of a contraction $C_{0}$-semigroup on $\overline{R(C)}$ ? If this holds, then $\left(\lambda-A^{\prime}\right)^{-1} \overline{R(C)}=\overline{R(C)}$ for all $\lambda>0$. Hence it is crucial that $\overline{R(C)}$ be an invariant subspace for $(\lambda-A)^{-1}$ since $A^{\prime} \subseteq A$. So one way to answer the question in the negative is to give a contraction $C$-semigroup with generator $A$, in

Received by the editors October 16, 2006, and, in revised form, February 12, 2008

2000 Mathematics Subject Classification. Primary 47D06; Secondary 47D03.

$K e y$ words and phrases. $C$-semigroups, contraction, $C_{0}$-semigroups, extrapolation space.

The first author was supported by the NSF of China (Grant No. 10501032), and the second author by TRAPOYT and the NSF of China (Grant No. 10671079). 
which $(\lambda-A)^{-1}$ does not leave $\overline{R(C)}$ invariant. It is easier to construct a bounded $C$-semigroup than a contraction one. Now the extrapolation space is helpful. By making use of it, we can obtain contraction $C$-semigroups from bounded ones.

Throughout this paper, for an operator $A$ on $X$, we write $D(A)$ for its domain, $R(A)$ for its range, and the closure of $R(A)$ is denoted by $\overline{R(A)}$. The $C$-resolvent set of $A, \rho_{C}(A):=\{\lambda \in \mathbb{C}: \lambda-A$ is injective and $R(C) \subset R(\lambda-A)\}$, and the $C$-resolvent of $A$ is $R_{C}(\lambda, A):=(\lambda-A)^{-1} C$ for $\lambda \in \rho_{C}(A)$. For $Y$ a subspace of $X$ and $A$ a linear operator on $X$, we denote by $\left.A\right|_{Y}$ the part of $A$ in $Y$, i.e., $\left.A\right|_{Y} \subset A$ with maximal domain. For the properties of $C$-semigroups and of contractions, we refer to 2, 4.

\section{MAin Results}

First we give a positive answer to the question mentioned above under some additional assumptions. The following result also improves Theorem 3.4 in [4.

Theorem 2.1. Let $A=C^{-1} A C, \overline{C D(A)}=\overline{R(C)}$ and $D(A) \subseteq R(r-A)$ for some $r>0$. Then the following are equivalent:

(a) A generates a contraction $C$-semigroup on $X$.

(b) $(0, \infty) \subseteq \rho_{C}(A)$ and $\lambda\left\|R_{C}(\lambda, A) x\right\| \leq\|C x\|$ for $\lambda>0$ and $x \in X$.

(c) $\left.A\right|_{\overline{R(C)}}$ generates a contraction $C_{0}$-semigroup on $\overline{R(C)}$.

Proof. $(a) \Rightarrow(b)$ follows from Theorem 3.3 in [4].

(b) $\Rightarrow(c)$. Define $B \subseteq A$ with $D(B)=C D(A)$. Then $B$ is a densely defined closable operator on $\overline{R(C)}$. By $(b),\|(\lambda-A) x\| \geq \lambda\|x\|$ for $\lambda>0$ and $x \in D(B)$; i.e., $B$ is dissipative. This implies that $\bar{B}$ is also dissipative and $R(\lambda-\bar{B})$ is a closed subspace of $\overline{R(C)}$. To show that $R(\lambda-\bar{B})=\overline{R(C)}$, let $x \in D(A)$. Since $D(A) \subseteq R(r-A), x=(r-A) y$ for some $y \in D(A)$ and $A C y=C A y$ due to the assumption $A=C^{-1} A C$,

$$
C x=(r-A) C y=(r-B) C y \in R(r-\bar{B}) .
$$

This implies that $\overline{R(C)}=\overline{C D(A)} \subseteq R(r-\bar{B})$, as desired. It now follows from the Lumer-Phillips theorem that $\bar{B}$ generates a contraction $C_{0}$-semigroup on $\overline{R(C)}$. It remains to show that $\bar{B}=\left.A\right|_{\overline{R(C)}}$. It is clear that $\left.\bar{B} \subseteq A\right|_{\overline{R(C)}}$, and so $\overline{R(C)} \subseteq$ $R\left(r-\left.A\right|_{\overline{R(C)}}\right)$. Also, the injectivity of $r-A$ implies that of $r-\left.A\right|_{\overline{R(C)}}$. Thus, $\bar{B}=\left.A\right|_{\overline{R(C)}}$ follows from the identity that $(r-\bar{B})^{-1}=\left(r-\left.A\right|_{\overline{R(C)}}\right)^{-1}$.

$(c) \Rightarrow(a)$. Let $T(t)=S(t) C$, where $S(t)$ is the contraction $C_{0}$-semigroup generated by $\left.A\right|_{\overline{R(C)}}$ on $\overline{R(C)}$. It is easy to show that $T(t)$ is a contraction $C$ semigroup; we only need to show that $A$ is the generator. If $x \in D(A)$, then since $A C x=C A x \in R(C)$ by the assumption that $A=C^{-1} A C$, we know that $C x \in D(A \mid \overline{R(C)})$ and

$$
\frac{T(t) x-C x}{t}=\left.\frac{S(t) C x-C x}{t} \rightarrow A\right|_{\overline{R(C)}} C x=\left.C A\right|_{\overline{R(C)}} x=C A x
$$

as $t \rightarrow 0$, so an extension of $A$ is the generator. Suppose that $\lambda>0$; if $(\lambda-A) x=0$, then, since $C x \in D(A \mid \overline{R(C)})$,

$$
\left(\lambda-\left.A\right|_{\overline{R(C)}}\right) C x=(\lambda-A) C x=C(\lambda-A) x=0 .
$$


Thus $x=0$; i.e., $\lambda-A$ is injective. Also, for $x \in X$, let $y=R\left(\lambda,\left.A\right|_{\overline{R(C)}}\right) C x$. Then $C x=\left(\lambda-\left.A\right|_{\overline{R(C)}}\right) y=(\lambda-A) y$. This implies that $R(C) \subseteq R(\lambda-A)$ and so $\lambda \in \rho_{C}(A)$. Then it follows from Corollary 3.12 in [2] that $C^{-1} A C=A$ is the generator.

Now we turn to the construction of the extrapolation space. For simplicity, we only consider bounded $C$-semigroups.

Let $\{T(t)\}_{t \geq 0}$ be a bounded $C$-semigroup on $X$ with generator $A$, so there exists some constant $M>0$ such that $\|T(t)\| \leq M$ for all $t \geq 0$. For each $x \in X$, define $\|x\|_{s}=\sup _{t \geq 0}\|T(t) x\|$. Then

$$
\|C x\| \leq\|x\|_{s} \leq M\|x\| .
$$

Since $C$ is injective, $\|\cdot\|_{s}$ is a norm on $X$. Denote by $X_{s}$ the completion of $X$ with respect to the norm $\|\cdot\|_{s}$. Extend $T(t)$ to $X_{s}$ by defining $T_{s}(t) y=\lim _{n \rightarrow \infty} T(t) x_{n}$ for all $t \geq 0$, with the limit taken in $X$, whenever $\left\{x_{n}\right\}$ is a sequence in $X$ converging to $y$, in $X_{s}$. We also denote by $C_{s}$ the extension of $C$ to $X_{s}$. It is not hard to see that $T_{s}(t)$ is bounded on $X_{s}$ for each $t \geq 0$, and $C_{s}$ is injective.

Theorem 2.2. Let $X_{s}, T_{s}(t), C_{s}$ be as above. Then

(a) For all $t \geq 0, R\left(T_{s}(t)\right)$ is contained in $\overline{R(T(t))}$, the closure of $R(T(t))$ in $X$. In particular, $R\left(T_{s}(t)\right) \subseteq X$ and $R\left(C_{s}\right) \subseteq \overline{R(C)}$, the closure of $R(C)$ in $X$.

(b) $\left\{T_{s}(t)\right\}_{t \geq 0}$ is a strongly uniformly continuous contraction $C_{s}$-semigroup.

(c) Suppose that $A_{s}$ is the generator of $\left\{T_{s}(t)\right\}_{t \geq 0}$. Then

( $\left.c_{1}\right) A \subseteq A_{s}$;

(c) $A_{s}=C_{s}^{-1} A C_{s}$;

(c $\left.c_{3}\right) A=\left.A_{s}\right|_{X}$.

Proof. (a) follows immediately from the definition of $T_{s}(t)$.

(b). First, we show that $T_{s}\left(t_{1}+t_{2}\right) C_{s}=T_{s}\left(t_{1}\right) T_{s}\left(t_{2}\right)$ for all $t_{1}, t_{2} \geq 0$. Let $y \in X_{s}$. Then there exists $\left\{x_{n}\right\} \subset X$ such that $x_{n}$ converges to $y$ in $X_{s}$, which means that $T(t) x_{n}$ converges in $X$ for all $t \geq 0$. Also, by the definition of $T_{s}(t)$ and (a), we have

$$
\begin{aligned}
C_{s} T_{s}\left(t_{1}+t_{2}\right) y & =C \lim _{n \rightarrow \infty} T\left(t_{1}+t_{2}\right) x_{n}=\lim _{n \rightarrow \infty} C T\left(t_{1}+t_{2}\right) x_{n} \\
& =\lim _{n \rightarrow \infty} T\left(t_{1}\right) T\left(t_{2}\right) x_{n}=T\left(t_{1}\right) \lim _{n \rightarrow \infty} T\left(t_{2}\right) x_{n} \\
& =T\left(t_{1}\right) T_{s}\left(t_{2}\right) y=T_{s}\left(t_{1}\right) T_{s}\left(t_{2}\right) y
\end{aligned}
$$

with the four limits taken in $X$.

Next, for every $x \in X$,

$$
\left\|T_{s}(t) x\right\|_{s}=\|T(t) x\|_{s}=\sup _{r \geq 0}\|T(r) T(t) x\|=\sup _{r \geq 0}\|T(r+t) C x\| \leq\|C x\|_{s}=\left\|C_{s} x\right\|_{s}
$$

therefore, $\left\{T_{s}(t)\right\}_{t \geq 0}$ is a family of contractions since $X$ is dense in $X_{s}$.

Finally, we show that $\left\{T_{s}(t)\right\}_{t \geq 0}$ is strongly uniformly continuous. Now let $y \in X_{s}$. Then there exists a sequence $\left\{x_{n}\right\} \subset X$ satisfying $\left\|x_{n}-y\right\|_{s} \rightarrow 0$ as 
$n \rightarrow \infty$. Thus

$$
\begin{aligned}
& \left\|T_{s}(t+h) y-T_{s}(t) y\right\|_{s} \\
\leq & \left\|T_{s}(t+h) y-T_{s}(t+h) x_{n}\right\|_{s}+\left\|T_{s}(t+h) x_{n}-T_{s}(t) x_{n}\right\|_{s} \\
& \quad+\left\|T_{s}(t) x_{n}-T_{s}(t) y\right\|_{s} \\
\leq & 2\left\|C_{s}\left(x_{n}-y\right)\right\|_{s}+\sup _{r \geq 0}\left\|T(t+r+h) C x_{n}-T(t+r) C x_{n}\right\| \\
\leq & 2\left\|C_{s}\left(x_{n}-y\right)\right\|_{s}+M\left\|T(h) x_{n}-C x_{n}\right\| .
\end{aligned}
$$

We already use the contractivity of $T_{s}(t)$ in the above. Note that the right side is independent of $t$, so $\left\{T_{s}(t)\right\}_{t>0}$ is strongly uniformly continuous.

$\left(c_{1}\right)$. Suppose that $x \in D(A)$. Then by (2.1), we know

$$
\begin{aligned}
\left\|\frac{T_{s}(t) x-C_{s} x}{t}-C_{s} A x\right\|_{s} & =\left\|\frac{T(t) x-C x}{t}-C A x\right\|_{s} \\
& \leq M\left\|\frac{T(t) x-C x}{t}-C A x\right\| \rightarrow 0 \text { as } t \rightarrow 0 ;
\end{aligned}
$$

it follows that $x \in D\left(A_{s}\right)$ with $A_{s} x=A x$.

$\left(c_{2}\right)$. If $y \in D\left(A_{s}\right)$, then $\left\|\frac{T_{s}(t) y-C_{s} y}{t}-C_{s} A_{s} y\right\|_{s} \rightarrow 0$ as $t \rightarrow 0$. Since $R\left(T_{s}(t)\right) \subseteq$ $X$, by the definition of $\|\cdot\|_{s}$, we have

$$
\left\|\frac{T(h)\left(T_{s}(t) y-C_{s} y\right)}{t}-T(h) C_{s} A_{s} y\right\| \rightarrow 0 \text { as } t \rightarrow 0
$$

uniformly in $h$. Set $h=0$. Noting that $C_{s}$ commutes with $A_{s}$ and $T_{s}(t)$, we have

$$
\left\|\frac{T_{s}(t) C_{s} y-C_{s} C_{s} y}{t}-C_{s} A_{s} C_{s} y\right\| \rightarrow 0 \text { as } t \rightarrow 0 .
$$

Since $C_{s} y \in D\left(A_{s}\right) \cap X$ and $A_{s} C_{s} y=C_{s} A_{s} y \in X$, this means

$$
\left\|\frac{T(t) C_{s} y-C C_{s} y}{t}-C A_{s} C_{s} y\right\| \rightarrow 0 \text { as } t \rightarrow 0,
$$

which implies that $C_{s} y \in D(A)$ and $A C_{s} y=A_{s} C_{s} y=C_{s} A_{s} y$, i.e., $A_{s} y=$ $C_{s}^{-1} A C_{s} y$. So we get $A_{s} \subseteq C_{s}^{-1} A C_{s}$.

On the other hand, $C_{s}^{-1} A C_{s} \subseteq C_{s}^{-1} A_{s} C_{s}=A_{s}$ since $A_{s}$ is the generator.

$\left(c_{3}\right)$. If $x \in D\left(A_{s}\right) \cap X$ and $A_{s} x \in X$, then $C x=C_{s} x \in D(A)$ by (b) and $A C x=A_{s} C x=C_{s} A_{s} x=C A_{s} x$, which implies that $A_{s} x=C^{-1} A C x$. So the claim follows from the fact that $A=C^{-1} A C$.

Remark 2.3. (a) It should be mentioned that the extrapolation space, $W$, of [1, 2, is defined only when $R(C)$ is dense; in [3] it is defined when $R(C)$ is dense or $\rho(A)$ contains a half-line. When $R(C)$ is dense, generating a contraction $C$-semigroup is equivalent to generating a strongly continuous semigroup of contractions by Theorem 4.6 in [4]; thus $A_{s}$ of Theorem 2.2 is such a generator when $R(C)$ is dense.

(b) Recall the definition of $W$ in [1] or [2]: for $x \in X,\|x\|_{W}=\sup _{t \geq 0}\|T(t) x\|=$ $\|x\|_{s}$. Since $W$ is a Banach space containing $X$, and $X_{s}$ is the completion of $X$ under the norm $\|\cdot\|_{s}$, it is clear that $X_{s}$ is contained in $W$ when $R(C)$ is dense and $W$ is defined.

(c) $T_{s}(t)$ from Theorem 2.2 is a nonincreasing $C$-semigroup:

$$
\left\|T_{s}(r) x\right\|_{s}=\sup _{t \geq 0}\|T(t) T(r) x\|=\sup _{t \geq r}\|T(t) C x\|,
$$


which is nonincreasing as a function of $r$. This implies that $e^{r A}$, at least formally a strongly continuous semigroup generated by $A$, is a contraction on $\bigcup_{t \geq 0} R(T(t))$, defined by $e^{r A} T(t) x \equiv T(t+r) x$.

(d) As a consequence of (c), when $\bigcup_{t>0} R(T(t))$ is dense, $A_{s}$ of Theorem 2.2 generates a strongly continuous semigroup of contractions. This is a weaker hypothesis than $R(C)$ being dense.

Now we use the extrapolation space to give a negative answer to the question mentioned in the Introduction.

Example 2.4. Let $X=c_{0}(\mathbb{N})$ and $C_{0}$ be the right shift on $X$, that is,

$$
C_{0}:\left(x_{1}, x_{2}, x_{3} \cdots\right) \rightarrow\left(0, x_{1}, x_{2}, x_{3}, \cdots\right) \text {. }
$$

Next let

$$
A=\left(\begin{array}{cc}
i & C_{0}^{-1} \\
0 & -i
\end{array}\right) \text { with } D(A)=X \times R\left(C_{0}\right)
$$

and

$$
C=\left(\begin{array}{cc}
C_{0} & 0 \\
0 & C_{0}
\end{array}\right)
$$

It is not hard to show that $A$ generates a bounded $C$-semigroup on $X \times X$ given by

$$
T(t)=\left(\begin{array}{cc}
e^{i t} C_{0} & \frac{1}{2 i}\left(e^{i t}-e^{-i t}\right) \\
0 & e^{-i t} C_{0}
\end{array}\right),
$$

but $\{T(t)\}_{t \geq 0}$ is not contractive. For every $\lambda \neq 0$, if $x_{2} \notin \overline{R\left(C_{0}\right)}$, then

$$
(\lambda-A)^{-1} C\left(\begin{array}{c}
x_{1} \\
x_{2}
\end{array}\right)=\left(\begin{array}{c}
(\lambda-i)^{-1} C_{0} x_{1}+(\lambda+i)^{-1}(\lambda-i)^{-1} x_{2} \\
(\lambda+i)^{-1} C_{0} x_{2}
\end{array}\right) \notin \overline{R(C)} .
$$

So $(\lambda-A)^{-1}$ does not leave $\overline{R(C)}$ invariant. Since $\left\|C_{0} x\right\|=\|x\|$, for all $x \in X$, so $X_{s}=X$, and $\|\cdot\|_{s}$ is a topologically equivalent renorming of $X$. Thus $T_{s}(t)=T(t)$, $(\lambda-A)^{-1}=\left(\lambda-A_{s}\right)^{-1}$. Therefore $\left(\overline{R\left(C_{s}\right)}\right)_{s}$ is not an invariant space of $\left(\lambda-A_{s}\right)^{-1}$. Thus no restriction of $A_{s}$ generates a contraction $C_{0}$-semigroup on $\left(\overline{R\left(C_{s}\right)}\right)_{s}$.

Remark 2.5. (a) The result is true for any injective $C_{0} \in \mathbf{B}(X), X$ an arbitrary complex Banach space, satisfying $\overline{R\left(C_{0}\right)} \neq X$ and $0 \notin \sigma_{a}\left(C_{0}\right)$; i.e., $C_{0} x_{n} \rightarrow 0$ implies $x_{n} \rightarrow 0$.

(b) Although $A_{s}$ of Example 2.4 does not generate a strongly continuous semigroup on $\overline{R(C)}$, there does exist a subspace, $Y$, between $\overline{R(C)}$ and $X_{s}$, on which $A_{s}$ generates a strongly continuous semigroup, namely, $Y=X \times \overline{R\left(C_{0}\right)}$.

We end this paper with some open questions:

1. Is every contraction $C$-semigroup a nonincreasing $C$-semigroup (meaning $t \mapsto\|T(t) x\|$ is nonincreasing, for all $x \in X)$ ? This is true for $C$ being isometric; that is, $\|C x\|=\|x\|$ for all $x \in X$, since in this case,

$\|T(t+s) x\|=\|C T(t+s) x\|=\|T(t+s) C x\|=\|T(t) T(s) x\| \leq\|C T(s) x\|=\|T(s) x\|$.

We conjecture that it is not true in general cases.

2. If $A$ generates a contraction $C$-semigroup on $X$, does there exist a closed subspace $Y$ such that $\overline{R(C)} \subseteq Y \subseteq X$ and $\left.A\right|_{Y}$ generates a strongly continuous semigroup of contractions? Example 2.4 of this paper shows that the answer is no if $Y$ is replaced by $R(C)$, but as remarked above in the section on Example 2.4, the answer is yes (in Example 2.4) with a different choice of $Y$. 
3. Does every generator of a bounded $C$-semigroup have an extension, possibly on a larger space, that generates a strongly continuous semigroup of contractions? If 2 is true, then it and Theorem 2.2 would imply the answer is yes; when $R(C)$ is dense or $\rho(A)$ contains a half-line, it is known $([1,2,3])$ that the answer is yes.

4. Is there a minimal Banach space in which $X$ is embedded on which an extension of $A$ generates a bounded, strongly continuous semigroup? Even when $R(C)$ is dense, so that an extension as in 3 exists, it is not known if a minimal one exists. In contrast, the interpolation space is maximal (see Chapter V in 2]).

5. Does $X_{s}=W$ always (when $R(C)$ is dense, so that both are defined)? Or is there an example where $W$ is strictly larger than $X_{s}$ ?

\section{ACKNOWLEDGEMENT}

We are very grateful to the referees for some improvement of the results, a simplification of Example 2.4 and for addressing the last five open questions.

\section{REFERENCES}

1. R. deLaubenfels, C-semigroups and strongly continuous semigroups, Israel J. Math. 81 (1993), 227-255. MR.1231189 (95d:47047)

2. R. deLaubenfels, Existence Families, Functional Calculi and Evolution Equations, Lecture Notes in Math. 1570, Springer-Verlag, Berlin, 1994. MR.1290783 (96b:47047)

3. V. Keyantuo, Interpolation and extrapolation of $C$-semigroups, Publ. Math. Fac. Sci. Besançon, Anal. Nonlinéaire 12 (1990), 103-121.

4. M. Li and F.L. Huang, Characterizations of contraction C-semigroups, Proc. Amer. Math. Soc. 126 (1998), 1063-1069. MR1443839 (98f:47048)

Department of Mathematics, Sichuan University, Chengdu, Sichuan 610064, People's Republic of China

E-mail address: limiao1973@hotmail.com

Department of Mathematics, Huazhnog University of Science and Technology, Wuhan 430074, People's Republic of China

E-mail address: qzheng@hust.edu.cn 